\title{
Ansätze zur Analyse historischer Netzwerke mit Neo4j® - Aus der Projekt-Werkstatt der Datenbank zur Fachgeschichte der Musikwissenschaft
}

\author{
Annette van Dyck-Hemming, Jan Eberhardt, Melanie Wald-Fuhrmann, \\ Frankfurt a. M.
}

DOI: $10.25366 / 2020.107$

Zitation: Annette van Dyck-Hemming, Jan Eberhardt, Melanie Wald-Fuhrmann, „Ansätze zur Analyse historischer Netzwerke mit Neo4j® - Aus der Projekt-Werkstatt der Datenbank zur Fachgeschichte der Musikwissenschaft", in: Brückenschläge zwischen Musikwissenschaft und Informatik. Theoretische und praktische Aspekte der Kooperation, in Verbindung mit der Fachgruppe Digitale Musikwissenschaft hrsg. von Stefanie Acquavella-Rauch, Andreas Münzmay und Joachim Veit (= Musikwissenschaft: Aktuelle Perspektiven. Bericht über die Jahrestagung der Gesellschaft für Musikforschung 2019 in Paderborn und Detmold, Bd. 3), Detmold, Musikwissenschaftliches Seminar der Universität Paderborn und der Hochschule für Musik Detmold, 2020, S. 191-198, DOI: 10.25366/2020.107

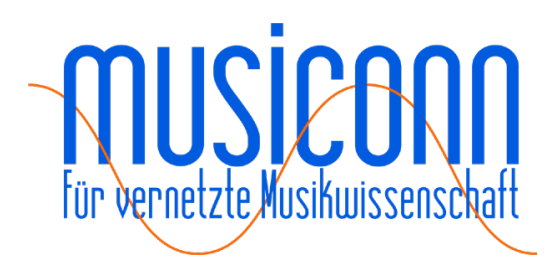




\title{
Ansätze zur Analyse historischer Netzwerke mit Neo4j ${ }^{\circledR}-$ Aus der Projekt-Werkstatt der Datenbank zur Fachgeschichte der Musikwissenschaft
}

\author{
ANNETTE VAN DYCK-HEMMING, JAN EBERHARDT, MELANIE WALD-FUHRMANN, \\ FRANKFURT A. M.
}

\section{Projektrahmen | Fragestellung}

Als Teil des Projektes "Fachgeschichte der deutschsprachigen Musikwissenschaft zwischen ca. 1810 und ca. 1990" werden in einer Datenbank Daten zu Personen, Institutionen, Medien, Themen und Methoden der Musikwissenschaft gesammelt. Der Fokus in der Modellierung liegt dabei auf der qualitativ angereicherten Beschreibung von Beziehungen zwischen diesen Entitäten.

Auf dieser Grundlage lassen sich eine ganze Reihe von Aspekten der Geschichte der Musikwissenschaft quantitativ ausdrücken, wodurch üblichere Verfahren historischer Quellenauswertung eine interessante Ergänzung erfahren können. Zu einem solchen Aspekt gehören z. B. personelle Netzwerke. Wir führen das im Folgenden vor am Beispiel einer Visualisierung von Daten zu den Personen Carl Dahlhaus und Hans Heinrich Eggebrecht. Beide Persönlichkeiten prägten vor allem die westdeutsche Musikwissenschaft zwischen den 1960er und 1990er Jahren sehr stark: Dahlhaus (1928-1989), Professor für historische Musikwissenschaft an der TU Berlin seit 1967, veröffentlichte etwa das Handbuch der Systematischen Musikwissenschaft; seine Publikationen zu Musikästhetik, den Grundlagen der Musikgeschichte, zur Geschichte der Musiktheorie uvm. wurden breit rezipiert. Hans Heinrich Eggebrecht (1919-1999) wirkte ab 1961 als Professor für Musikwissenschaft an der Universität Freiburg im Breisgau, edierte etwa das Handwörterbuch der musikalischen Terminologie und verfasste das umfangreiche monographische Geschichtswerk Musik im Abendland. Trotz ihrer wiederholten Zusammenarbeit z. B. als Herausgeber einer Neuauflage des Riemann Musik-Lexikons hat sich in der Musikwissenschaft die Ansicht verbreitet, Dahlhaus und Eggebrecht stellten zwei geradezu entgegengesetzte Pole dar, um die sich je eigene Netzwerke bildeten. Unsere Datenbank bietet nun die Möglichkeit, eine größere Anzahl von Beziehungsdaten im Hinblick auf diese These abzufragen. Die Visualisierung mittels der Graphdatenbank Neo4j ${ }^{\circledR 1}$ bildet das Ergebnis plastisch ab und lädt ein zur Interpretation: Wird die im Fach verbreitete Meinung gestützt oder konterkariert?

$1 \mathrm{Neo4j} \circledast$ and Cypher ${ }^{\circledR}$ are registered trademarks of Neo4j, Inc., <www.neo4j.com> (19.09.2019). 


\section{Quellen | Daten| Methoden}

\section{Datenauswahl}

Als Quellen wurden die MGG Online, ${ }^{2}$ Titeleien und Inhaltsverzeichnisse der Festschriften für Dahlhaus (1988) ${ }^{3}$ und Eggebrecht (1984) ${ }^{4}$ sowie die Bibliographie des Musikschrifttums Online (BMS Online) $)^{5}$ herangezogen. Aus diesen Quellen wurden folgende Daten unter Verwendung der jeweiligen Suchmöglichkeiten vollständig, aber qualitativ bereinigt (z. B. fanden Personennamen als Verlagsbezeichnungen keine Berücksichtigung) extrahiert:

- Namensnennungen in den Artikeln "Carl Dahlhaus" und "Hans Heinrich Eggebrecht" aus der MGG Online,

- Namensnennungen "Dahlhaus" oder „Eggebrecht" (nur Nachnamen) in Personenartikeln der MGG Online außerhalb von Literaturangaben (Zeitgenossen),

- Empfängernamen aller Artikel von Dahlhaus und Eggebrecht, die für Festschriften verfasst wurden,

- Namen aus den bibliographischen Angaben aller Publikationskooperationen

- Namen aus Titeleien und Inhaltsverzeichnissen der Festschriften Eggebrecht 1984 und Dahlhaus 1988

Immer zwei Namen wurden entsprechend den Quellen in Beziehung gesetzt. Dabei interessierten für diesen Werkstatt-Bericht nur sogenannte "professionelle Kooperationen" zwischen Personen und ihre nähere Beschreibung in Bezug auf Zeit und Ort, also nicht, mit wem jemand verheiratet oder befreundet war, auch wenn diese Informationen zur Verfügung standen. Die Beziehungsbeschreibungen entstanden unterschiedlich: Aus den biographischen Artikeln wurden die Formulierungen im Text so eng wie möglich übernommen („leitete Umarbeitung gemeinsam mit"). Hatte sich z. B. jemand im Rahmen einer Festschrift engagiert, wurde genau dies näher beschrieben, etwa als Beitrag („trägt zur Festschrift bei von“) oder als Herausgabe ("gibt Festschrift heraus von") etc.

\section{Datenstrukturierung}

Alle Daten wurden in eine relationale SQL-Datenbank eingepflegt, deren Datenmodell allerdings dem Resource Description Framework (RDF) bestehend aus semantischen Triples ähnelt,

2 MGG Online, hrsg. von Laurenz Lütteken, Kassel, Stuttgart, New York 2016ff., <www.mgg-online.com> (19.09.2019).

3 Hermann Danuser, Silke Leopold, Norbert Miller und Helga de La Motte-Haber (Hrsg.), Das musikalische Kunstwerk. Geschichte - Ästhetik - Theorie. Festschrift Carl Dahlhaus zum 60. Geburtstag, Laaber 1988.

4 Werner Breig, Reinhold Brinkmann und Elmar Budde (Hrsg.), Analysen. Beiträge zu einer Problemgeschichte des Komponierens. Festschrift für Hans Heinrich Eggebrecht zum 65. Geburtstag, Stuttgart 1984 (= Beihefte zum Archiv für Musikwissenschaft 23).

5 BMS Online, hrsg. vom Staatliches Institut für Musikforschung, Stiftung Preußischer Kulturbesitz, Berlin 2006ff., <https://www.musikbibliographie.de/> (19.09.2019). 
da es Entitäten durch prädikative Formulierungen in Relation setzt und die entstehenden „Aussagen" mit Attributen anreichert.

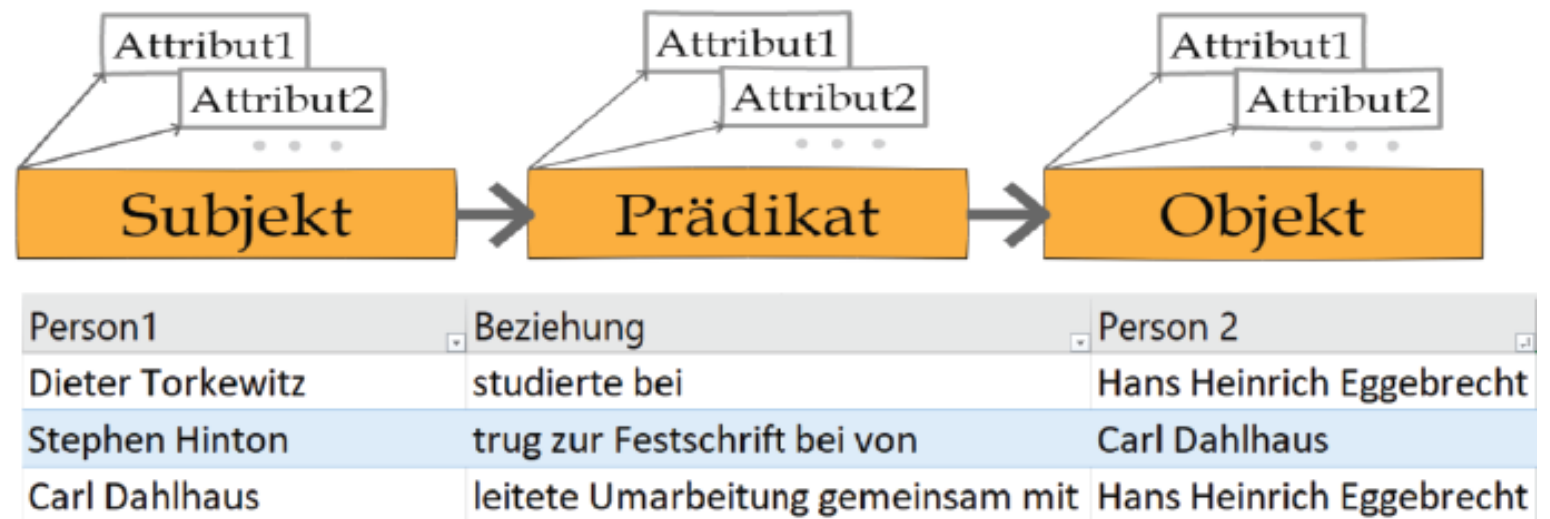

Abbildung 1: „Semantische" Modellierung der Daten

Anders als im RDF sind aber nicht alle Daten in semantischen Triples organisiert, sondern nach wie vor in Tabellen, wenn auch teilweise sehr kleinen Tabellen. Jeder Datensatz ist dabei mit einem Quellennachweis versehen.

\section{Datenbankabfrage und Visualisierung}

Die Datenbankabfrage wurde eingegrenzt auf den Zeitraum zwischen 1960 und 1990, auf Datensätze, die entweder den Namen Dahlhaus oder den Namen Eggebrecht enthielten, sowie auf Datensätze, die Beziehungen zwischen Personen beschreiben. Daraus resultierten 178 Datensätze.

Die Visualisierung erfolgte mittels der Graphdatenbank Neo4j ${ }^{\circledR}$. Hierzu wurden die Datensätze in der Abfragesprache Cypher ${ }^{\circledR}$ codiert und mit dem in der Neo4j ${ }^{\circledR}$ Desktop ${ }^{\mathrm{TM}}$ Anwendung enthaltenen Visualisierungsmodul als Netzwerk abgebildet (vgl. Abb. 2).

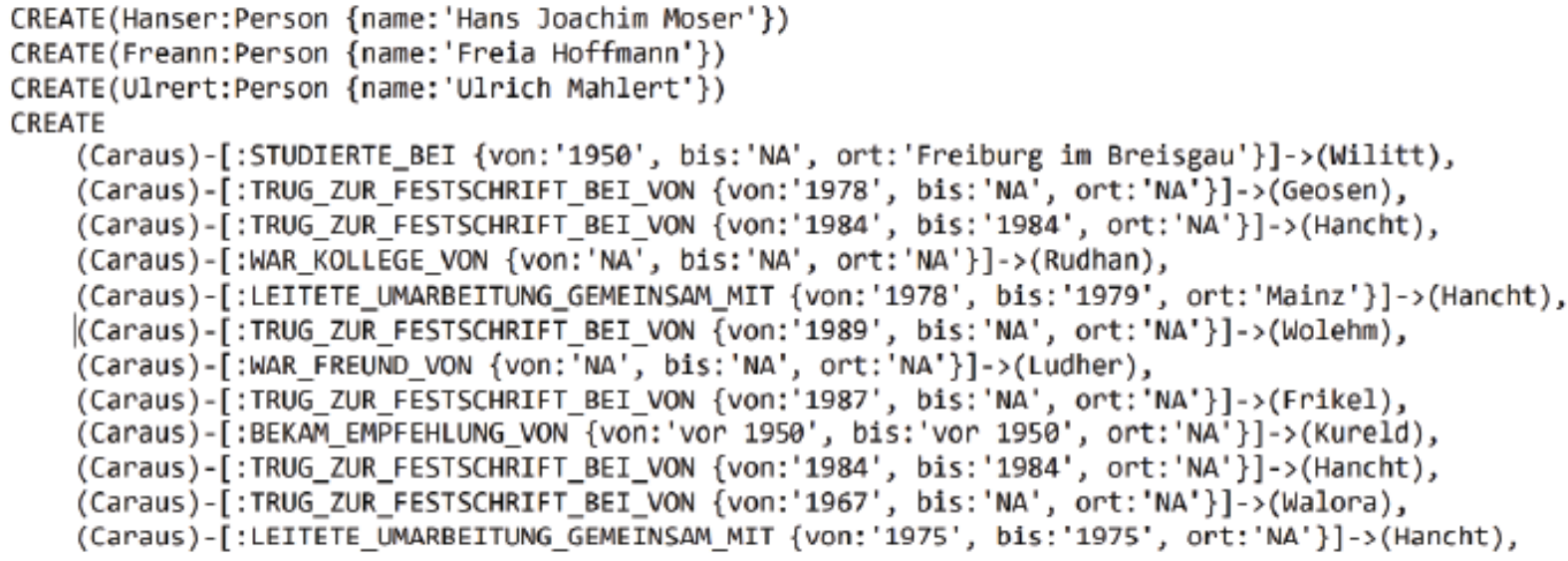

Abbildung 2: Ausschnitt aus der Codierung der Daten in der Abfragesprache Cypher ${ }^{\circledR}$ 
Das Neo4j ${ }^{\circledR}$ Visualisierungsmodul stellt alle als Person definierten Daten als Knoten (nodes)/ Kreise dar und beschriftet die Kanten (relationships) zwischen den Knoten mit der Beziehungscodierung. Details zu den Beziehungen werden in Kontextmenüs am Fensterrand angezeigt und quantifiziert (vgl. Abb. 3).

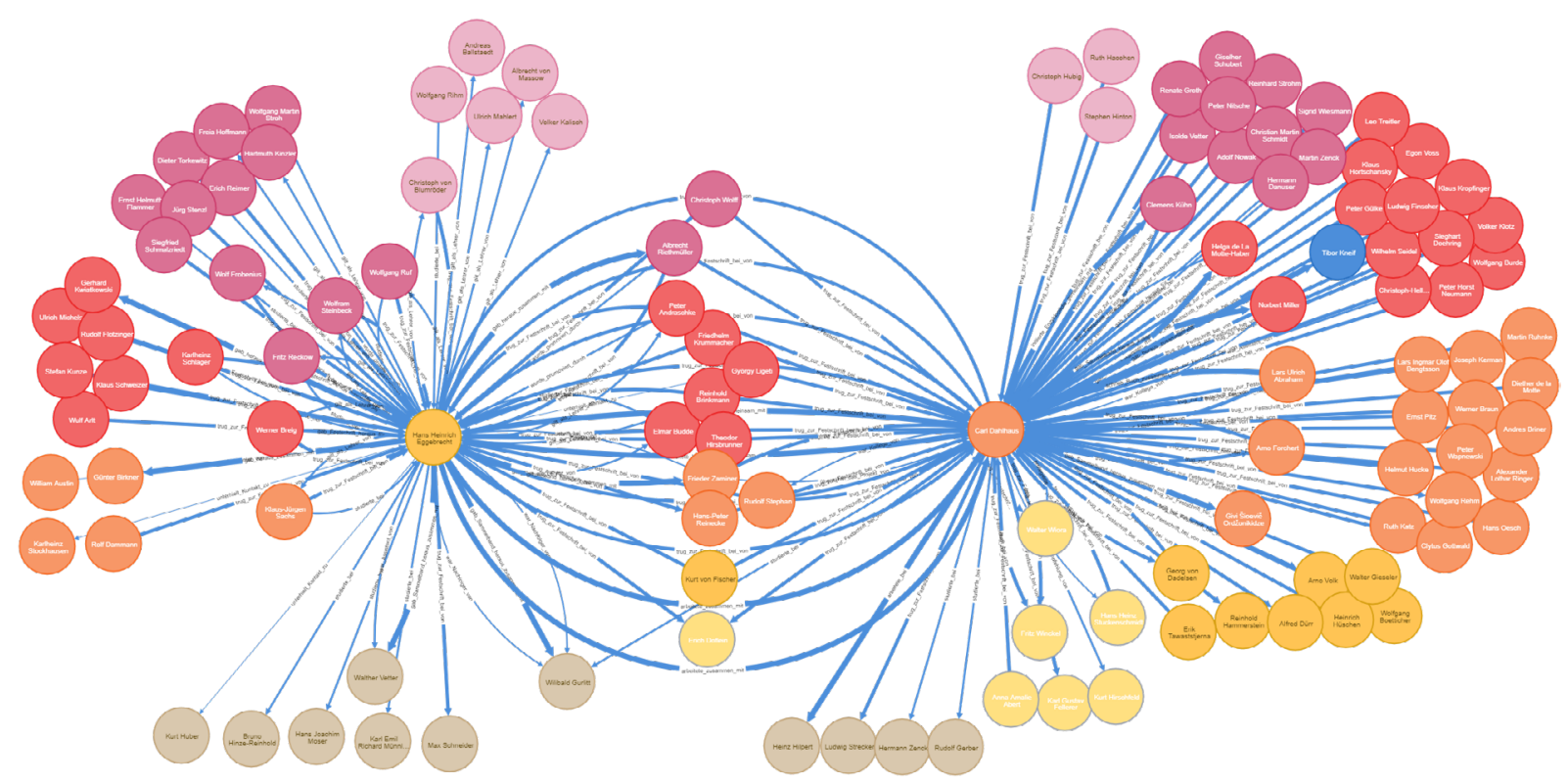

Abbildung 3: Bearbeitete Visualisierung mittels der Neo4j® Graphdatenbank ohne Legende

Die als Teil der Neo4j® Graphdatenbank angebotene plastische Visualisierung markiert den Punkt, an dem Argumentation und Interpretation der Forschenden anfangen. Die Platzierung der Knoten und die Gestaltung der Verbindungen zwischen ihnen wurden in diesem Anwendungsbeispiel manuell vorgenommen.

\section{Diskussion | Interpretation}

115 verschiedene Personen sind visuell um die beiden Zentren Dahlhaus und Eggebrecht geordnet. Die von der Software zunächst alle auf die gleiche Distanz gesetzten Akteure wurden so platziert, dass Akteure mit Beziehungen zu beiden Zentren in der Mitte stehen. Im Verhältnis ältere Personen stehen unten, jüngere oben. Akteure mit mehreren Beziehungen zu einem der Zentren wurden näher zu den Zentren geordnet. Zeitintensivere Beziehungen wie "leitete Umarbeitung zusammen mit" wurden durch breitere Verbindungen dargestellt als vermutlich weniger intensive Beziehungen wie "unterhielt Kontakt zu". Zur Mittelgruppe gehören wenige, nämlich 14 Personen. Dieser Darstellung zufolge prägten sich die personalen Netzwerke von 
Dahlhaus und Eggebrecht also nicht komplett dichotomisch aus. Auffällig sind die mehrfach definierten (stabilen?) Verbindungen Eggebrechts zu deutlich jüngeren Personen ("Schülern”), während das Dahlhaus-Netzwerk Mehrfach-Beziehungen eher zu gleichaltrigen oder älteren Personen aufweist. Um den jüngeren Dahlhaus zentrieren sich deutlich mehr Akteure mit einfachen Beziehungen. Eklatant scheint der Befund, dass Eggebrecht fast keine professionellen Beziehungen zu Mitgliedern seiner eigenen Generation unterhielt.

Wenn auch mit Blick auf die begrenzte Datenmenge Vorsicht geboten ist, könnte man dennoch Thesen und daran anschließende weiterführende Fragen formulieren: Tatsächlich sammelte jede der Leitfiguren einen je eigenen Kreis um sich. Die Schnittmenge ist stark begrenzt. Die Generationenverteilung scheint eine für den relativ geringen Altersunterschied der Protagonisten von 10 Jahren deutliche Rolle zu spielen: Wollte Eggebrecht mit Mitgliedern seiner eigenen Generation nichts zu tun haben? Weitere Fragen könnten durch Datenanreicherung oder weitere Überprüfungen beantwortet werden: Welche Gemeinsamkeiten weisen die Akteure der Schnittmenge auf? Welche Rolle spielen gemeinsame Forschungsschwerpunkte oder institutionelle Verbindungen? Wie würde sich die Darstellung verändern bei der Einbeziehung weiterer Daten, etwa der Beziehungen der 115 Akteure untereinander, der Zuordnung von Forschungsschwerpunkten etc.?

Eine Datenbankabfrage ist wie ein Magnet: In eine große Menge an sich eher unbedeutender Teilchen geworfen, erzeugt sie Bilder, die Regeln gehorchen. Die Art der Regeln und der Ausnahmen herauszufinden, ist die Aufgabe des/der Werfenden. Im Unterschied zum Magnetismus sind die Regelwerke personaler Netzwerke aber stärker faktorenabhängig, vielfältig und komplex. Flexible Visualisierungen von Beziehungsdaten etwa unter Verwendung einer Graphdatenbank wie Neo4j ${ }^{\circledR}$ können helfen, größere Datenmengen anschaulich darzustellen, Interpretationen zu entwickeln und Thesen zu Regelhaftigkeiten zu überprüfen und zu modifizieren.

\section{Literatur}

Ludwik Fleck, Entstehung und Entwicklung einer wissenschaftlichen Tatsache. Einführung in die Lehre vom Denkstil und Denkkollektiv, Basel 1935, hrsg. von Lothar Schäfer und Thomas Schnell, Frankfurt a. M. ${ }^{10} 2015$

Bruno Latour, Reassembling the Social. An Introduction to Actor-Network-Theory, Oxford 2005, dt. Frankfurt a. M. ${ }^{3} 2014$

Melanie Wald-Fuhrmann und Annette van Dyck-Hemming, „Vom Datum zum historischen Zusammenhang. Möglichkeiten und Grenzen einer fachgeschichtlichen Datenbank", in: Wissenskulturen der Musikwissenschaft: Generationen - Netzwerke - Denkstrukturen, hrsg. von Sebastian Bolz, Moritz Kelber, Ina Knoth und Anna Langenbruch, Bielefeld 2016, S. 261-278 
Ute Lemm, Musikwissenschaft in Westdeutschland nach 1945, Bonn 2005

Bernd Schirpenbach, Ästhetische Regulation und hermeneutische Überschreibung. Zum Begriff und zur musikwissenschaftlichen Funktion einer korrelativen Hermeneutik im Ausgang von Interpretationsund Wissenschaftskonzeptionen bei Dahlhaus und Eggebrecht, Stuttgart 2006

Jan Philipp Sprick, „Kann Musiktheorie ,historisch' sein?”, in: Zeitschrift der Gesellschaft für Musiktheorie Sonderausgabe: Musiktheorie | Musikwissenschaft. Geschichte - Methoden - Perspektiven (2010), S. 145-164, <https://doi.org/10.31751/597> (19.09.2019)

Zitation: Annette van Dyck-Hemming, Jan Eberhardt, Melanie Wald-Fuhrmann, „Ansätze zur Analyse historischer Netzwerke mit Neo4j® - Aus der Projekt-Werkstatt der Datenbank zur Fachgeschichte der Musikwissenschaft", in: Brückenschläge zwischen Musikwissenschaft und Informatik. Theoretische und praktische Aspekte der Kooperation, in Verbindung mit der Fachgruppe Digitale Musikwissenschaft hrsg. von Stefanie Acquavella-Rauch, Andreas Münzmay und Joachim Veit (= Musikwissenschaft: Aktuelle Perspektiven. Bericht über die Jahrestagung der Gesellschaft für Musikforschung 2019 in Paderborn und Detmold, Bd. 3), Detmold, Musikwissenschaftliches Seminar der Universität Paderborn und der Hochschule für Musik Detmold, 2020, S. 191-198, DOI: 10.25366/2020.107 


\section{Abstract}

The dichotomy Carl Dahlhaus-Hans Heinrich Eggebrecht appears to be legendary for West German musicology. It has been quite common to assume that since the 1960s and even after Dahlhaus' death in 1989, musicologists have been very strongly oriented towards these two persons, but actually only either Dahlhaus or Eggebrecht. But can such a legend be verified in information technology? How, if necessary, can one grasp and make understandable academic networks?

We have searched the entire MGG-Online for references to the names of Dahlhaus and Eggebrecht, we looked at Festschriften dedicated to Dahlhaus or Eggebrecht with regard to the persons who are marked in the texts/books as important for the two professors (also 'pupils', assistants, students etc.). All professional cooperations manifested through publications were also considered, including persons whose texts were edited by Dahlhaus or Eggebrecht. These data were converted into the format of a graph database and visualized.

The resulting graph shows a polarized network but a small group with connections to both lead figures, too. Eggebrecht's network reveals a remarkable gap regarding connections to members of his own generation. Dahlhaus' personal network seems to be significantly bigger but less intense regarding the relation quality. The findings exemplify the possibilities and limitations of the evaluation of historical data by graph database technologies.

\section{Kurzviten}

Melanie Wald-Fuhrmann ist Direktorin der Abteilung Musik am Max Planck-Institut für empirische Ästhetik in Frankfurt am Main. Sie wurde 2005 an der Universität Zürich mit einer Arbeit über Athanasius Kircher promovierte und habilitierte dort 2009 über Melancholie in der Instrumentalmusik um 1800. Sie war Professorin für Musikwissenschaft in Lübeck und für Musiksoziologie und historische Anthropologie der Musik an der Humboldt-Universität zu Berlin. Sie gibt das Lexikon Schriften über Musik und die Reihe Spektrum Fachgeschichte Musikwissenschaft heraus.

Annette van Dyck-Hemming koordiniert seit 2014 als Musikwissenschaftlerin das Projekt zur Fachgeschichte der deutschsprachigen Musikwissenschaft am Max Planck-Institut für empirische Ästhetik. Sie studierte an den Universitäten in Bochum (Magistra artium) und Bonn (Promotion über die Musik Elliott Carters). Bis 2012 organisierte sie die Neuedition des Riemann Musik Lexikons auf Herausgeberseite. 


\section{Ansätze zur Analyse historischer Netz- werke mit Ne04jß - Aus der Projekt-Werkstatt der}

\section{Datenbank für Fachgeschichte der Musikwissenschaft}

\section{Projektrahmen | Fragestellung}

Als Teil des Projektes 'Fachgeschichte der deutschsprachigen Musikwissenschaft zwischen ca. 1810 und ca. 1990' werden in einer Datenbank Daten zu Personen, Institutionen, Medien, Themen und Methoden der Musikwissenschaft gesammelt. Der Fokus in der Modellierung liegt dabei auf der qualitativ angereicherten Beschreibung von Beziehungen zwischen diesen Entitäten. Auf dieser Grundlage lassen sich dann eine ganze Reihe von Aspekten der Geschichte der Musikwissenschaft quantitativ ausdrücken, wodurch üblichere Verfahren historischen Quellenauswertung eine interessante Ergänzung erfahren können. Ein solcher Aspekt sind z. Bsp. personelle Netzwerke. Wir führen das im Folgenden vor am Beispiel einer Visualisierung von Daten zu den Personen Carl Dahlhaus und Hans Heinrich Eggebrecht. Es steht außer Frage, dass beide die deutsche Musikwissenschaft zwischen den 1960er und 1990er Jahren stark prägten. Trotz ihrer wiederholten Zusammenarbeit hat sich aber die Ansicht von zwei geradezu entgegengesetzten Polen verbreitet um die sich je eigene Netzwerke bildeten. Unsere Datenbank bietet nun die Möglichkeit, eine größere Anzahl von Beziehungsdaten abzufragen. Die Visualisierung mittels der Graphdatenbank Neo4j® bildet das Ergebnis plastisch a und lädt ein zur Interpretation: Wird die im Fach verbreitete Meinung gestützt oder konterkariert?

Datenstrukturierung

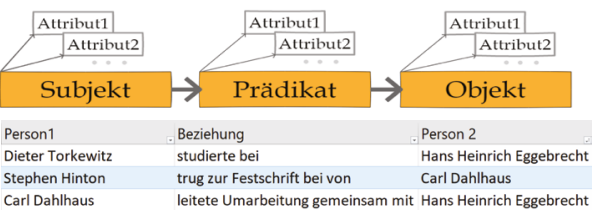

Die Struk tischen Triples, ist aber Teil (a)

Alle Namen referieren auf GND-Normdaten. Zu allen Datensätzen sind
Quellen angegeben.

\section{Quellen | Daten| Methoden}

1. Datenauswahl

- Namesnennungen in den biografischen Artikeln von Dahlhaus und Eggebrecht aus der MGG Online

Namensnennungen Dahlhaus oder Eggebrecht in Personenartikeln in der MGG Online außerhalb von Lit.angaben (Zeitgenossen),

Empfängernamen aller Artikel von Dahlhaus und Eggebrecht, die sie für Festschriften verfasst haben,

Namen aus bibliogr. Angaben aller Publikationskooperationen

Namen aus Titeleien und Inhaltsverzeichnissen der Festschriften Eggebrecht 1984 und Dahlhaus 1988

- in Texten beschriebene Beziehungen (nur professionelle Kooperationen) zwischen Personen inkl. Attributen Zeit und Ort, wenn vorhanden

- vollständig (Recherchegrundlage BMS Online), aber bereinigt: z. B. keine Berücksichtigung von Personennamen als Verlagsbezeichnungen

2. Datenstrukturierung für eine relationale Datenbank, aber RDF-ähnlich

3. Datenbankabfrage in SQL und Codierung in der Abfragesprache Cypher®

- Zeitraum zwischen 1960 und 1990, Datensätze mit den Namen Dahlhaus und Eggebrecht, nur Beziehungen zwischen Personen

->178 Datensätze

Code für Neo4j®

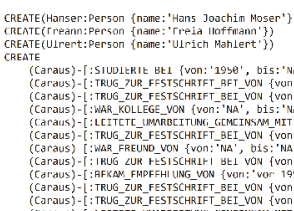

Mit Cypher® werden erst Stellvertreter für die Personen, dann die Beziehungen zwischen den Personen generiert.

- Die Beziehungsdefinition hat große struktuelle Ähnlichkeit zu den Rohdaten.

Ne04j® ist eine Graphdatenbank. Graphdatenbanken ermöglichen die (auch visuelle) Repräsentation komplexer Datenstrukturen.

\section{Das visuelle Ergebnis}

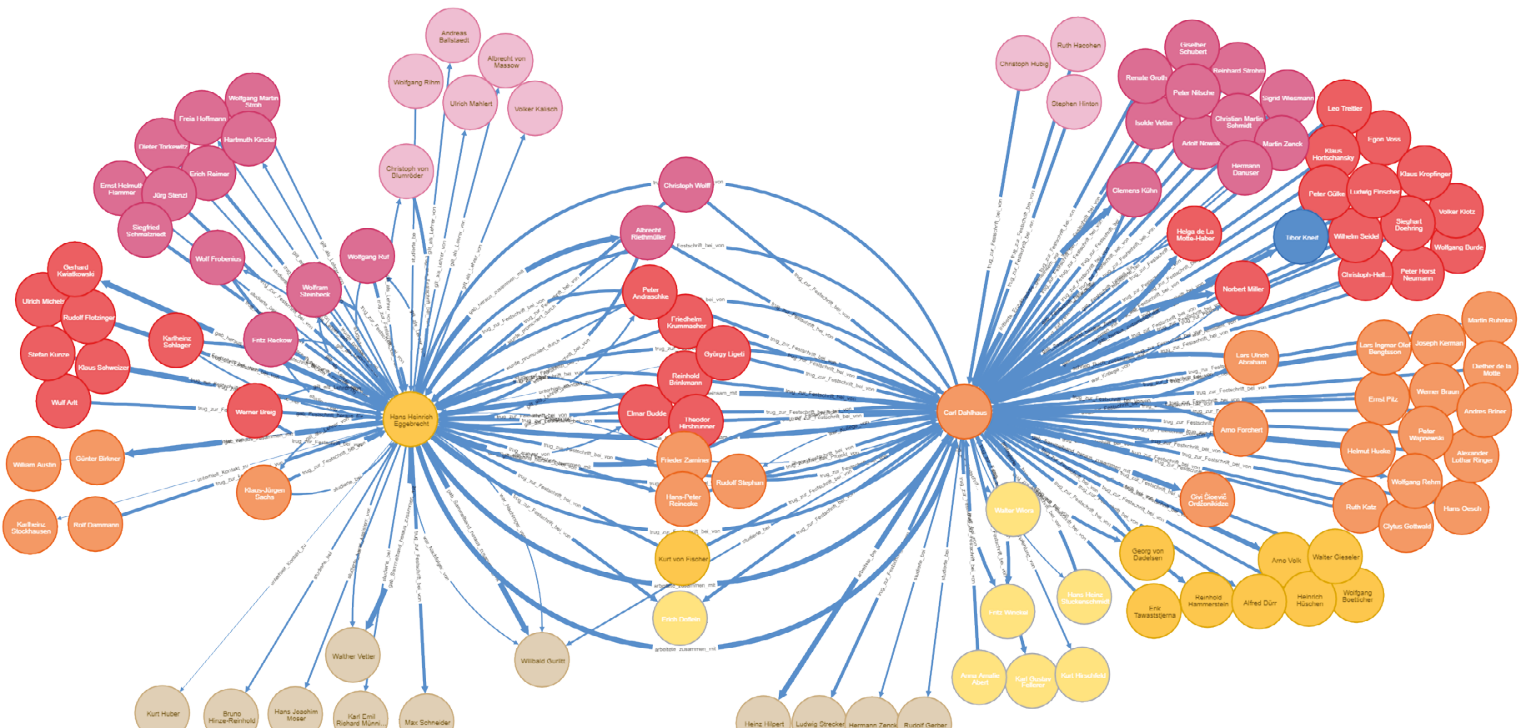

- Personen werden als Knotenpunkte (nodes)/Kreise dargestellt.

- Die Verbindungen (relationships) zwischen den Knoten werden korrekt beschriftet.

- Details zu den Beziehungen werden in Kontextmenüs am Fensterrand angezeigt.

\section{Diskussion | Interpretation | Ausblick, Bewertung}

117 verschiedene Personen sind visuell um die beiden Zentren Dahlhaus und Eggebrecht geordnet. Die vom Visualisierungsmodul der Graphdatenbank Neo4j® zunächst alle auf die gleiche Distanz gesetzten Akteure wurden so platziert, dass Akteure mit Beziehungen zu beiden Zentren in der Mitte stehen. Im Verhältnis ältere Personen stehen unten, jüngere oben. Akteure mit mehreren Beziehungen zu einem der Zentren wurden näher zu den Zentren geordnet. Zur Mittelgruppe gehören wenige, nämlich 14 Personen. Dieser Darstellung zufolge prägten seren Personen ('Schülern') während das Dahlhaus-Netzwerk Mehrfach-Beziehungen eher zu gleichaltrigen oder älteren Personen aufweist. Um den jüngeren Dahlhaus zentrieren sich gertlich mehr Akteure mit einfachen Beziehungen.

Vorsichtig und mit Blick auf die begrenzte Datenmenge könnte man dennoch Thesen formulieren: Tatsächlich ist festzustellen, dass jede der Leitfiguren einen eigenen Kreis um sich sammelte und die Schnittmenge hier doch stark begrenzt scheint. Die exklusive 'Mittelgruppe' ist interessant. Weisen ihre Mitglieder gemeinsame Eigenschaften/Zuordnungen auf? Die Generationenverteilung scheint eine fur den relativ geringen Altersunterschied der Protagonisten von 10 Jahren eine nicht zu unterschäzende Rolle zu spielen. Es bleiben Fragen offen, die man (115Akteure unte ander, der Zuordnung von Forschungsschwerpunkten etc.?

- Eine Datenbankabfrage ist wie ein Magnet: in eine große Menge an sich eher unbedeutender Teilchen geworfen, erzeugt sie Bilder, die Regeln gehorchen. Die Art der Regeln und der Ausnahmen herauszunden, ist die Aufgabe des/der Werfenden. Im Unterschied zum Magnetismus sind die Regelwerke personaler Netzwerke aber starker faktorenabhangig, vielfaltig und überprüfen, zu modifizieren und zu entwickeln.

\section{Kontakt}

Melanie Wald-Fuhrmann, Annette van

Dyck-Hemming | fachgeschich-
Literatur: Bruno Latour, Reassembling the Social. An Introduction to Actor-Network-Theory. Oxford 2005, dt. Frankfurt/M. 32014; Ludwik Fleck, Entstehung und Entwicklung einer wissenschaftlichen Tatsache. Einführung in die Lehre vom Denkstil und Denkkollektiv, Basel 1935, hrsg. von Lothar Schäfer und Thomas Schnell, Frankfurt/M. ${ }^{102015 ~-~ L e m m, ~ U t e, ~ M u s i k w i s s e n s c h a f t ~ i n ~ W e s t d e u t s c h l a n d ~ n a c h ~ 1945, ~ B o n n ~ 2005 ; ~ S c h i r p e n b a c h, ~ B e r n d, ~ A ̈ s t h e t i s c h e ~}$ Regulation und hermeneutische Überschreibung. Zum Begriff und zur musikwissenschaftlichen Funktion einer korrelativen Hermeneutik im Ausgang von Interpretations- und Wissenschaftskonzeptionen bei Dahlhaus und Eggebrecht, Stuttgart 2006; Sprick, Jan Philipp, " Kann Musiktheorie shistorischr sein?«, in: Zeitschrift der Gesellschaft für Musiktheorie Sonderausgabe (2010): Musiktheorie | Musikwissenschaft. Geschichte - Methoden - Perspektiven (2010), S. 145-164, https://doi.org/10.31751/597; Neo4j® and Cypher® are registered trademarks of Neo4j, Inc., https://neo4j.com/https://- 



\section{Brückenschläge zwischen Musikwissenschaft und Informatik}

Theoretische und praktische Aspekte der Kooperation

Herausgegeben von Stefanie Acquavella-Rauch,

Andreas Münzmay und Joachim Veit 
Brückenschläge zwischen Musikwissenschaft und Informatik 


\section{Musikwissenschaft: Aktuelle Perspektiven}

Bericht über die Jahrestagung der Gesellschaft für Musikforschung 2019 in Paderborn und Detmold

Herausgegeben von Rebecca Grotjahn und Nina Jaeschke

Band 3 


\section{Brückenschläge zwischen Musikwissenschaft und Informatik}

Theoretische und praktische Aspekte der Kooperation

Beiträge der Symposien zur Digitalen Musikwissenschaft

Osnabrück 2018 und Paderborn 2019

im Rahmen der Jahrestagungen der Gesellschaft für Musikforschung

In Verbindung mit der Fachgruppe Digitale Musikwissenschaft herausgegeben von

Stefanie Acquavella-Rauch, Andreas Münzmay und Joachim Veit

Detmold: Musikwissenschaftliches Seminar der Universität Paderborn und der Hochschule für Musik Detmold 2020 
DOI: $10.25366 / 2020.87$

Online-Version verfügbar unter der Lizenz: Urheberrecht 1.0, $<$ https://rightsstatements.org/page/InC/1.0/?language =de >

Bibliografische Information der Deutschen Nationalbibliothek

Die Deutsche Nationalbibliothek verzeichnet diese Publikation in der Deutschen Nationalbibliografie; detaillierte bibliografische Daten sind im Internet über http://dnb.d-nb.de abrufbar.

\section{Impressum}

Redaktion: Stefanie Acquavella-Rauch, Andreas Münzmay und Joachim Veit Satz: Nina Jaeschke und Joachim Veit

(C) Musikwissenschaftliches Seminar der Universität Paderborn und der Hochschule für Musik Detmold 2020 


\section{INHALT}

Rebecca Grotjahn, Nina Jaeschke

Vorwort zu Band 1-3

IX

Stefanie Acquavella-Rauch, Andreas Münzmay, Joachim Veit

Brückenschläge zwischen Musikwissenschaft und Informatik - Vorbemerkung

$\mathrm{XI}$

\section{KOLLABORATIONEN - KO-LABORATORIEN}

\section{Reinhard Keil}

Der Computer als Denkzeug für hermeneutische Arbeit

\section{Ulrich Konrad}

Philologie und Digitalität. Perspektiven für die Musikwissenschaft im Kontext fächerübergreifender Institutionen

\section{Gudrun Oevel}

Infrastruktureinrichtungen in Forschungsprojekten - Spagat oder Chance?

\section{Dennis Ried}

Erhebung, Transformation und Präsentation digitaler Forschungsdaten

Anna Neovesky, Frederic von Vlahovits

IncipitSearch - Leitfaden zur Zusammenarbeit

Elisabeth Treydte

Clara Schumann \#digital. 40 Jahre Archiv Frau und Musik und der Start in die Digitalisierung

\section{TEXT/DATEN/PROZESSE}

\section{Christine Siegert}

Komponisten-Gesamtausgaben im digitalen Zeitalter: Perspektiven

und Reflexionen am Beispiel Ludwig van Beethovens

Markus Neuwirth, Johannes Hentschel, Martin Rohrmeier

Perspectives of Musical Corpus Studies: The Annotated Mozart Sonatas

Agnes Amminger, Franz Kelnreiter

Leopold Mozarts „Gründliche Violinschule". Zur Textcodierung und -präsentation einer digitalen Edition

\section{Oleksii Sapov}

Algorithmische Automatisierung komplexer Notationsregeln in MEI-XML am Beispiel von Versetzungszeichen 


\section{Susanne Cox, Richard Sänger}

Digitale Fassungsvergleiche am Beispiel von Beethovens Eigenbearbeitungen

Agnes Seipelt

Digitale Edition und Harmonische Analyse mit MEI von Anton Bruckners

Studienbuch

Stefanie Acquavella-Rauch

Musikalische Schaffensprozesse 2.0 - Inkorporation audiovisueller Medien

der populären Musik in Methoden der digitalen Edition

\section{DIGITAL(ISIERT)E MATERIALITÄTEN}

\section{Miriam Akkermann}

(Musik)Instrument (im) Computer

\section{Daniel Fütterer}

Herausforderungen bei der Kodierung von Paratext am Beispiel Neuer Musik mit Live-Elektronik

\section{Matthias Pasdzierny}

How much is the glitch? Das digitale Paradigma als Herausforderung

und Chance für die historische Musikwissenschaft

\section{Shintaro Miyazaki}

Musik für Maschinen?! - Wo sich die Wissenschaft der Medien, des Computers und der Musik treffen und wie sie zusammenarbeiten könnten

\section{MUSIKGESCHICHTE(N) IM NETZ}

\section{Matthias Tischer}

Musikgeschichte der DDR: Ein Pilotprojekt zur digitalen Musikvermittlung

Annette van Dyck-Hemming, Jan Eberhardt, Melanie Wald-Fuhrmann

Ansätze zur Analyse historischer Netzwerke mit Neo4j® - Aus der Projekt-Werkstatt der Datenbank zur Fachgeschichte der Musikwissenschaft

Axel Beer, Martin Bierwisch, Kristina Krämer

Das MMM2 - Ein regionalgeschichtliches Onlinelexikon der Arbeitsgemeinschaft für mittelrheinische Musikgeschichte

\section{Matej Santi}

Was erzählt Fritz Kreislers Geige?

\section{Elias Berner}

Alle Menschen werden Brüder?! Ein historisches Dokument aus dem

Nationalsozialismus in den sozialen Medien 
Gabriele Buschmeier in memoriam 